\title{
26. DISCUSSION FOLLOWING MEZGER'S REPORT
}

\author{
(Thursday, September 18, 1969)
}

\section{Chairman: I. S. SHKLOVSKII}

Editor's remark: The Discussion has been rearranged into five sections: 1. Density Concentrations, Cocoon Stars, and OH Sources; 2. The Carbon Recombination Line; 3. The He/H Abundance; 4. Various Shorter Comments; 5. A New Mechanism for Star Formation. A part of the Discussion which related to Spiegel's Report has been transferred to Chapter 13. A short exchange on $\mathrm{HI}$ shells around $\mathrm{H}$ II regions has been deleted and added to Chapter 5. A short part on the dust cloud in Orion at the end of Section 4 has been transferred from Tuesday, September 16.

\section{Density Concentrations, Cocoon Stars, and OH-Sources}

Menon: Dr. Mezger, I recall that in one of your early papers with Burke and others you refer to a new class of $\mathrm{HII}$ regions, called compact $\mathrm{H}$ II regions, which you identified as cocoon stars. Now you called the Orion Nebula a compact Hil region. Are these two objects the same?

Mezger: More observations with high angular resolution have led us to revise our ideas of the nature of compact $\mathrm{H}$ II regions. I now believe that most of the compact Hil regions with linear dimensions of less than $0.5 \mathrm{pc}$, which we observe with an angular resolution of $2^{\prime}$, are the ionized remnants of a protocluster rather than of a protostar. Perhaps in the case of very young objects, like DR $21^{*}$ or G133.9+1.1* in IC 1795, we are seeing the ionized shells of very young individual $O$ stars. In most other cases the compact $\mathrm{H}$ II regions are already more expanded and of lower average density than cocoon stars. Often the regions contain a number of condensations, part of which may be protostars of lower mass. If one wants to see these individual condensations in extended compact $\mathrm{H}$ II regions, one has to use higher angular resolution, such as the aperture synthesis maps of the Orion Nebula, of M17, of W49, and of IC 1795 recently obtained by Webster and Altenhoff (1970; see also Figure 2 in the preceding chapter). To answer your question concerning Orion: not the Orion Nebula but its two components G209.0-19.4 (M42) and G208.9-19.3 (M43) are considered to be compact H II regions. (Webster, W. J. and Altenhoff, W.: 1970, Astrophys. Lett. 5, 233.)

Verschuur: I do not see how this concept of cocoon stars originated. How do you know that the star is surrounded by dust? The only thing you can tell is that, as seen from Earth, the star is behind the dust.

* DR 21 is source No. 21 found in the study of the Cygnus X region by D. Downes and R. Rinehart (1966, Astrophys. J. 144, 937). The symbol G133.9 + 1.1 stands for a source with galactic (G) coordinates $l=133^{\circ} .9, b=+1^{\circ} .1$. (Ed.) 
Mezger: Schraml and I investigated about $20 \mathrm{HII}$ regions with very high surface brightness at $\lambda=2 \mathrm{~cm}$ (Schraml and Mezger, 1969). About one-half of the sources can be optically identified, but in many cases (e.g., the component G133.7+1.2 in IC 1795) we find that the centers of radio emission do not coincide with anything seen in the optical range. In all optically identified Hil regions, with the exception of the Orion Nebula, C. B. Stephenson, who is one of the most experienced observers of $O$ stars, searched for the exciting stars but could not find them. Only in two cases did he find highly-reddened OB stars. This shows that the exciting stars are obscured. But also in OB star associations which are associated with young Hil regions, e.g., the Cepheus IV OB star association or M16, we do not seem to observe enough exciting stars. This can be shown as follows: the radio continuum flux from an Hil region is directly related to the Lyman continuum flux of the exciting stars. In most Hil regions, and especially in compact, young Hil regions, it turns out that the Lyman continuum flux of the visible stars is not sufficient to explain the radio continuum flux and that there must be some additional sources of ionization, such as for example, stars hidden behind a dust cocoon. (Schraml, J. and Mezger, P. G.: 1969, Astrophys. J. 156, 269).

Verschuur: That is no answer. Why cannot the stars just be behind the dust? Why do you conclude that they are surrounded by dust?

Mezger: Well, in the case of the 07 star Herschel 36, in M8, you really see the obscuring dust cloud around the star which is embedded in the Hil region with two very bright nebulosities, known as the Hourglass Nebula, at the western rim of the dust cloud.

Verschuur: It is two-dimensional. You are concluding something about three dimensions.

Mezger: It would be rather strange that the dust cloud with a diameter of $18^{\prime \prime}$ just happens to coincide with the star. On a more statistical basis you may consider Reddish's dust-embedded stars in young associations as an indication that most O stars are surrounded by dust clouds.

Let me mention one more piece of information on dust clouds in $\mathrm{H}$ II regions. Apart from extinction of light, which gives an indirect method of observing dust clouds, there is a direct method through thermal radiation in the far IR. The mechanism is simple: the dust grains are heated by starlight and then re-radiate in the IR. Typical temperatures of the dust grains appear to be a few hundred $\mathrm{K}$, thus their IR radiation is best observed at 10 or $20 \mu$. Extended IR nebulae have first been observed northwest of the Trapezium stars in the Orion Nebula by Kleinmann and Low (1967); subsequently a second IR nebula, approximately coincident with the Trapezium stars, has been discovered by Ney and Allen (1969). In the Hil region M17, observed at radio wavelengths, two compact radio sources have been found after observations with an angular resolution of $2^{\prime}$, and at the VIth Liège Symposium on Pre-main Sequence Stellar Evolution Low reported that he observed two extended IR nebulae coinciding with the radio components. He further claimed to have observed two stars, which by his IR measurements he can identify as $\mathrm{O}$ stars located within the boundaries of the two IR nebulae. This is further strong evidence for the existence of obscuring 
dust clouds in HII regions which surround the exciting stars. How the stars manage to ionize the gas through these dust cocoons is, however, not yet clear. (Kleinmann, D. E. and Low F. J.: 1967, Astrophys. J. Lett. 149, L1; Ney, E. D. and Allen, D. A.: 1969, Astrophys. J. Lett. 155, L193.)

Pottasch: At present I would not yet draw the conclusion that emission from the dust causes the IR nebulae observed by Low. Nothing is known about the spectra of these objects; other radiation mechanisms are conceivable that would explain the observations. I think that $\mathrm{H}$ i gas alone would certainly be enough to produce it. The work of Stein and Gillett (1969a) is perhaps applicable, but is not at all conclusive. (Stein, W. A. and Gillett, F. C.: 1969, Astrophys. J. Lett. 155, L197.)

Mezger: I don't think that is correct, since the spectrum of at least the infrared nebula in Orion has been investigated by Stein and Gillett (1969). They find a bump in the spectrum between 9 and $12 \mu$ which they claim can best be explained by emission of mineral dust grains. That is, in fact, how the silicate grains came into play.

Shklovskii: Does the presence of $\mathrm{OH}$ sources in an $\mathrm{H}$ II region correlate with other characteristics of $\mathrm{H}$ II regions?

Menon: The $\mathrm{OH}$ sources in the Orion Nebula could not have been detected in the present surveys, if the Nebula had been twice as distant. So, all Hil regions might have similar sources of anomalous $\mathrm{OH}$ emission.

Weaver: Strength of source is certainly an important factor in the discovery of anomalous $\mathrm{OH}$ emission regions; many faint sources must remain to be found. However, there may be an additional factor.

So far anomalous $\mathrm{OH}$ sources have been detected only in very dirty HiI regions, that is, in H II regions that are closely associated with large amounts of dark material. It may be that only in $\mathrm{H}$ II regions embedded in dark material are conditions appropriate for the production of anomalous $\mathrm{OH}$ sources.

Verschuur: Dr. Mezger, do you imply in your discussion on the $\mathrm{OH}$ point sources in W3 (=IC 1795), that there are seven protostars, or that these are all aspects of one protostar, because they are after all, within a few thousand AU of one another.

Mezger: That is a good question. In view of their different radial velocities I do not believe that all seven emission centers pertain to one protostar. However, the double and triple sources which form one emission center might probably be part of one protostar.

Menon: Some time ago I used the argument, again in connection with the Orion Nebula $\mathrm{OH}$ sources, that the velocity range of the components is very large, of the order of $30 \mathrm{~km} \mathrm{sec}^{-1}$ or more. If all sources are situated within less than $0.1 \mathrm{pc}$, as indicated by measurements, their lifetime in terms of separation from each other is less than a few thousand years. So, whatever the detailed nature of the objects might be, the time scale for the phenomenon is only a few thousand years, and the sources could not all be together in such a small space, unless they had been formed a very short time ago. This argument is, I think, still valid.

Mezger: I might add, though, that in discussing the protostar hypothesis Robinson and I (Mezger and Robinson, 1968) did not work from the assumption that $\mathrm{OH}$ 
emission centers are protostars and then tried to find out whether or not the conditions in both types of objects are compatible. It was just the other way around. Making some assumptions, which might be bad or good, we tried to infer the densities, the masses, and the temperatures of $\mathrm{OH}$ emission centers. It turned out that the temperatures and the densities agree with those in protostars, but the masses do not. But by assuming that either the maser effect is less effective or that only the outer layers of the protostar are involved in $\mathrm{OH}$ emission one gets to stellar masses. If this is true, however, the densities derived are so high that the emission centers must be gravitationally unstable and contract to form a star. (Mezger, P. G. and Robinson, B. J.: 1968, Nature 220, 1107.)

Burke: I hope that someone will tell me what a cosmic maser looks like. There are three models of cosmic masers. Since we have representatives of the protostar school present, we will call one model a protostar model. In this case, the condensed object is the entire maser. In W3 we are seeing, then, a cluster of young stars. The second model says that we are merely looking at filaments, accidentally end-on. In other words, one has a large region corresponding to the entire $\mathrm{W} 3$ region; all of this is in an inverted state. Here, there are no protostars at all. The third possible model one could call, I suppose, a condensation model of the maser. This is a model Gold proposed. One has a large region that is a potential maser. Accidental fluctuations cause a few regions to be stronger sources of emission than others. Then, when somewhere radiation is emitted, the outgoing spherical wave grows exponentially and eventually overtakes the inverse square law. Initially, you have many centers competing for a maser, but eventually one center dominates the masering region. Of the three possible models, I think that the observations throw some doubt upon the filamentary model. But the other two are still in the running. I wish to add one problem, which has to do with the tendency of the angular sizes of $\mathrm{OH}$ sources to grow with the distance of the sources from us. Take for example W3. It is reasonably close; its distance is $2 \mathrm{kpc}$. Its angular size is typically of the order of $0.01^{\prime \prime}$ for the individual features. With W49, however, its distance is more like $14 \mathrm{kpc}$; its angular size is of the order of $0.1^{\prime \prime}$. Thus it is farther away, larger in size. This may refer more to the conditions in the interstellar medium between the source and us, or it may mean that we have more than one kind of cosmic maser.

Mezger: As far as W49 is concerned, Dr. Burke is complaining about the fact that the angular sizes get larger in spite of the larger distance. However, I think one should keep in mind that the size of the $\mathrm{OH}$ emission centers in W49 corresponds precisely to the average distance of the individual emission sources found in W3. Could it be that your angular resolution was not sufficient to resolve the individual emission centers?

Burke: From the fringe visibility it is excluded that structure exists of a much smaller angular size.

\section{The Carbon Recombination Line}

Sorochenko: Dr. Mezger, what are the physical conditions in the regions where you observe the carbon lines? 
Mezger: From the line width we conclude that the temperature must be less than $1000 \mathrm{~K}$. Furthermore, the carbon line, when observed at different frequencies, behaves quite differently from the corresponding hydrogen lines. In the center of the Orion Nebula the carbon line has been observed at four wavelengths between $6 \mathrm{~cm}$ and $21 \mathrm{~cm}$ and its intensity relative to the hydrogen lines increases with increasing wavelength. This behavior suggests that the carbon lines are not emitted in LTE conditions and that some maser action is effective. This effect is by far not as spectacular as the maser amplification observed in the emission of $\mathrm{OH}$ and $\mathrm{H}_{2} \mathrm{O}$ lines and it may be explained by a weak dependence on $n$ of the $b_{n}$ factors, as has been first suggested by Goldberg.

Dyson: Would you give us the evidence for the conclusion that $\mathrm{C}^{0}$ is responsible for the recombination line?

Mezger: The conclusion is partly based on theoretical considerations. First, if the emission comes from neutral clouds, which are only partially ionized, then the ionization potential of the ionized atoms must be lower than that of hydrogen. If the emitter is one of the elements with high cosmic abundance only carbon is left. Second, this so-called carbon line is found in or close to nearly all Hil regions whose general radio recombination spectrum is intense enough. (To date the line has been found in all $\mathrm{HII}$ regions which have been investigated with sufficient signal-to-noise ratio.) On the assumption that this line is in fact the recombination line of carbon, it never has exactly the same radial velocity as the hydrogen recombination line at the center of the HII region. However, as far as we can say at present the observed deviations in radial velocities are random and not systematic. This is consistent with the idea that this line is in fact the recombination line of carbon and that it is emitted from a small region which has a slightly different radial velocity with respect to the central part of the Hil region.

Burke: I seem to remember that Goldberg uses one more argument, namely, that $\mathrm{C}^{+}$has a uniquely large dielectronic recombination cross section.

Mezger: I think dielectronic recombination is out, as far as Goldberg is concerned. Recently Mrs. A. K. Dupree, one of Goldberg's associates, has made more quantitative computations (Dupree, 1969). She found that the process of dielectronic recombination, if it is to result in a maser effect, first requires relatively high temperatures, much higher than the electron temperatures derived for normal Hil regions. Second, this maser amplification should show a very strong wavelength dependence, with a maximum amplification probably around 5 or $6 \mathrm{~cm}$. The observed increase of the intensity of the carbon line with increasing wavelength rules out an overpopulation by dielectronic recombination, as has been stated by Goldberg himself (1968). [Goldberg, L.: 1968, Interstellar Ionized Hydrogen (ed. by Y. Terzian and W. A. Benjamin), New York, p. 373; Dupree, A. K.: 1969, Astrophys. J. 158, 491.]

Sorochenko: Dr. Mezger, I have two questions about the carbon line. First, have calculations been made on the maser effect with realistic parameters and is there agreement between the theoretical calculation and the observed intensities? Second, when this line was observed. it was identified as a carbon line, because of the favorable 
dielectronic recombination rate. Since this argument seems to be invalid now are other elements becoming possibilities?

Mezger: As far as the first question is concerned, no; there are no computations of the $b_{n}$ factors for the physical conditions supposedly present in the emitting clouds. $R e$ question two: the observers did not base the identification of the line on the dielectronic recombination rate (Palmer et al., 1967); they used the fact that the line widths are so narrow that the kinetic temperatures of the emitting atoms must be less than $1000 \mathrm{~K}$. Thus we concluded that the radiation comes from cool, dense and only partly ionized clouds and consequently the ionization potential of the emitting atom must be lower than that of hydrogen. On the other hand, the emitter must be an abundant element. These were our original arguments, and only a subsequent paper by Goldberg and Dupree (1967) suggested dielectronic recombination as the cause for the maser effect, a theory which has been abandoned in the meantime, as mentioned before. (Goldberg, L. and Dupree, A. K.: 1967, Nature 215, 41 ; Palmer, P., Zuckerman, B., Penfield, H., Lilley, A. E., and Mezger, P. G.: 1967, Nature 215, 40).

Van Woerden: Do I understand correctly that the carbon lines come from an $\mathrm{HI}$ region?

Mezger: Yes, I think they originate in dense condensations, which are either imbedded in or located at the edge of an $\mathrm{HII}$ region. The condensations have densities of the order of $10^{5}$ or $10^{6}$ atom $\mathrm{cm}^{-3}$, and, even if exposed to the Lyman continuum radiation from a very early $\mathrm{O}$ star, they will be only superficially ionized. Thus, all the flux below $912 \AA$ will be absorbed in a thin surface layer but the radiation between 912 and $1100 \AA$, which can ionize $\mathrm{C}^{0}$, can freely penetrate the condensation.

Dyson: Will your comments regarding carbon remain valid, if you allow the possibility of ionization by cosmic rays?

Mezger: Why should cosmic rays single out one specific element?

\section{The $\mathrm{He} / \mathrm{H}$ Abundance}

Shklovskii: A very fundamental problem for cosmogonical studies is the abundance of He relative to H. Dr. Mezger, will you comment on this point?

Mezger: Measurements of He-recombination lines have been started by the Harvard observing group and me (Palmer et al., 1969), and have been pursued in the NRAOMIT and NRAO-CSIRO-MIT $109 \alpha$ recombination line surveys of the northern and southern sky (in press)* and most recently by Churchwell and me (in preparation).* We have investigated in detail something like $10 \mathrm{H} \mathrm{I}$ regions for their $\mathrm{He}$ to $\mathrm{H}$ abundance. For a much larger number of $\mathrm{HII}$ regions we have at least qualitative results. As I mentioned before, the measurement of the $\mathrm{He} / \mathrm{H}$ abundance, is rather straight-

* (Note added in proof.) References: Reifenstein, E. C., Wilson, T. L., Burke, B. F., Mezger, P. G., and Altenhoff, W. J.: 1970, Astron. Astrophys. 4, 357; Wilson, T. L., Mezger, P. G., Gardner, F. F., and Milne, D. K.: 1970, Astron. Astrophys. 6, 364; Churchwell, E. and Mezger, P. G.: 1970, Astrophys. Lett. 5, 227. 
forward, since the transition probabilities of the radio recombination lines for He and $\mathrm{H}$ are the same. Consequently, a comparison of the intensities immediately yields the number ratio. The present results can be summarized as follows.

(i) Most $\mathrm{HII}$ regions are located between $4 \mathrm{kpc}$ and $13 \mathrm{kpc}$ distance from the galactic center. This is the region where star formation occurs and spiral arms are observed. In this region, either no He-lines are observed at all, since the exciting stars are not hot enough (as in NGC 2024), or the observed He abundance is about $N\left(\mathrm{He}^{+}\right) / N\left(\mathrm{H}^{+}\right) \approx 0.08$. There are, however, two Hil regions (DR 21 and NGC 7538) which require early-type $\mathrm{O}$ stars for their ionization but nevertheless do not show He109 $\alpha$-emission.

(ii) There are about five 'giant HII regions' (i.e., whose intrinsic flux is equal or larger than four times the radio flux from the Orion Nebula) supposedly located inside the 4-kpc arm. Two out of these five Hil regions have been recently investigated by Churchwell and me and no He109 $\alpha$-emission could be detected (the upper limit was $\left.N\left(\mathrm{He}^{+}\right) / N\left(\mathrm{H}^{+}\right) \lesssim 0.02\right)$. However, we are not yet willing to interpret this as a true deficiency in $\mathrm{He}$ for the population II material in the center region of our Galaxy. These $\mathrm{H}$ II regions may have different excitation sources and for example the He could be doubly ionized. Therefore we first have to search for the recombination lines of doubly-ionized He before we can interpret the absence of the He109 $\alpha$-line.

(iii) The HII region 30 Doradus in the LMC shows an He abundance of 0.16 . Although the error margins on these observations are rather large, our observations (NRAO-CSIRO-MIT) are not compatible with a He abundance of 0.08 . There is a paper by Rubin (1968), who investigated the possibility of a non-coincidence of the Strömgren spheres of helium and hydrogen. His conclusion is that there are two possibilities: either the helium is more or less fully ionized within the Strömgren sphere of hydrogen, or it is not ionized at all. The transition range between these two states is very small; it occurs, according to Rubin's computations, at effective star temperatures of about $36000 \mathrm{~K}$. (Palmer, P., Zuckerman, B., Penfield, H., Lilley, A. E., and Mezger, P. G.: 1969, Astrophys. J., 156, 887; Rubin, R. H.: 1968, Astrophys. J. 153, 761.)

Ozernoi: The primeval $\mathrm{He} / \mathrm{H}$ ratio in the Universe is of great importance, as Shklovskii remarked. If the abundance of He should turn out to be low, this does not mean that the big-bang theory is incorrect. It means, perhaps, that the early stages of cosmological expansion are anisotropic or that processes are important which decrease the $\mathrm{He}$ abundance. In some quasars the $\mathrm{He}$ abundance is ten times smaller than usual, but at present it is unclear whether the lower abundance is due to anisotropic expansion of the Universe or to nonthermal processes in nuclei of quasars. In this connection, I would like to know: Is there any correlation between the regions of low $\mathrm{He}$ abundance and the sources of nonthermal radiation such as $\mathrm{OH}$ sources, infrared sources, supernovae, and the galactic center? [At this point there were some language problems. Part of Ozernoi's question is answered by the fact that recombination lines and $\mathrm{He} / \mathrm{H}$ abundances have been detected only in $\mathrm{HII}$ regions. (Ed.)] 
Mezger: Radio observations of the two Hil regions near the galactic center yield the following results. (i) The radio continuum spectrum appears to be thermal. (ii) The $\mathrm{H} 109 \alpha$ line-to-continuum ratio is that observed for typical spiral arm Hil regions corresponding to electron temperatures of less than $10000 \mathrm{~K}$. (iii) The shape of the $\mathrm{H} 109 \alpha$-lines, however, is rather odd, revealing either the existence of several components or of a very high internal turbulence. Although the two sources look pretty much like normal $\mathrm{HII}$ regions, the radio observations cannot rule out a source of ionization different from early-type stars. In this case it would not be unreasonable to expect that most of $\mathrm{He}$ is doubly ionized. I feel that only after observations of the $\mathrm{He}^{++}$-recombination line should we continue to speculate on the nature of these thermal sources inside the $4 \mathrm{kpc}$ arm.

Field: The other possibility that you mentioned is that the exciting stars are of spectral type later than $\mathrm{O} 9$ and that the $\mathrm{He}$ atoms are all $\mathrm{He}^{0}$. Is that not something we could also verify by computing the number of recombinations in the $\mathrm{HII}$ regions and checking that against the spectral type of the star?

Mezger: Yes, it could be done. Of course, you cannot decide from radio observations whether the ionization is caused by a number of B0 stars or by one $\mathrm{O} 5$ star. However, I feel it is reasonable to assume that all giant $\mathrm{H}$ II regions, i.e., those which have fluxes four times the flux of the Orion Nebula must be ionized by $\mathrm{O}$ stars. And these two Hil regions located close to the galactic center belong definitely to this class of giants.

Burke: I have only a quick comment on helium abundances. Not only in the galactic center are there $\mathrm{HII}$ regions that show very low helium abundances. Several other Hil regions (I think NGC 2264 is one of them) have quite low He abundances. There is even one that has an abundance of 3 per cent.

Mezger: To my knowledge in all the $\mathrm{H}$ II regions where we have been able to see the $\mathrm{He} 109 \alpha$-line at all, a $\mathrm{He} / \mathrm{H}$ ratio of 8 per cent is within the limits set by observational errors.

Field: Finally, there is the question of the excitation of the $\mathrm{He}$ lines. Is there not good evidence now from the study of the hydrogen recombination lines that we are dealing with maser effects, and that, therefore, these methods are unreliable for abundance determinations?

Mezger: First, we are doing experiments on the effects of deviations from LTE. I think all observers agree at the moment that deviations from LTE do not amount to more than a few times 10 per cent, if present at all. There are several observational studies possible. Let us consider, as an example, the $109 \alpha$ recombination spectrum. Within $6 \mathrm{MHz}$ one observes the H109 $\alpha$ line, the H137 $\beta$ line, the Hel09 $\alpha$ line, and the He $137 \beta$ line. [See Figure 3 at page 342 (Ed.)]. These four lines are observed simultaneously and a comparison of their intensities should yield considerable information on deviations from LTE and on the He abundances. Some of these observations have already been done, others are under way. Let me summarize our preliminary results. (i) In cases where the $\mathrm{H} 137 \beta$ line is too weak (as compared to the LTE case) the He137 $\beta$ line appears to be too weak by the same factor. This would 
indicate that $\mathrm{H}$ and $\mathrm{He}$ lines are affected by deviations from LTE in the same way. (ii) In the case of the Orion Nebula we have taken broadband spectrograms in the center as well as in four offset points spaced by one half the HPBW [= antenna beam (Ed.)] of the telescope. Although the ratio $\mathrm{H} 137 \beta / \mathrm{H} 109 \alpha$ for the two off-set points east and west of the center of the Orion Nebula is pretty close to the LTE-value, the measured $\mathrm{He} 109 \alpha / \mathrm{H} 109 \alpha$ ratio is, within the relatively small observational uncertainties, the same in all five points. (iii) We cannot find significant correlations between deviations from LTE (as measured by the ratio $\mathrm{H} 137 \beta / \mathrm{H} 109 \alpha$ ) and the He abundance (as determined from the ratio $\mathrm{He} 109 \alpha / \mathrm{H} 109 \alpha$ ). Further measurements of the $\mathrm{He} / \mathrm{H}$ ratio at higher and lower frequencies are under way.

Field: I was very interested in Ozernoi's suggestion that mechanisms might exist which destroy helium. Since such mechanisms might operate in nonthermal radio sources where cosmic rays are present, I was reminded of the fact that in the Crab Nebula supernova remnant the $\mathrm{He}$ abundance is anomalous. I would like to ask whether or not one can observe the He recombination lines in that Nebula to verify the anomaly.

Pottasch: I thought that in the Crab Nebula the helium abundance is at least twice as high as normal. That was Woltjer's (1958) result. (Woltjer, L.: 1958, Bull. Astron. Inst. Netherl. 14, 39.)

Mezger: As far as the observations are concerned, we have not found recombination lines in any nonthermal source. In general we have found many sources which do not show recombination lines, about 31 per cent for the northern sky, about 13 per cent for the southern sky. It appears that all of the sources which do not emit radio recombination lines have nonthermal continuum spectra. In fact in a few well-known supernova remnants we searched hard for radio recombination lines and did not find any. Either the electron temperatures are too low, and the lines are so wide, owing to high internal velocities, that they become unobservable, or the electron temperatures are too high, and the lines cannot be observed since they are too weak. To be quite specific, we observed the Crab Nebula and found no recombination lines whatsoever.

Field: And would you have expected them from calculations of electron density and temperature?

Mezger: A search for radio recombination lines in supernova remnants was suggested to us by Minkowski, who claims that the electron temperatures are low enough to observe the recombination lines. If the electron temperatures were in fact $10000 \mathrm{~K}$ in the Crab Nebula, it would be very easy for us to observe the lines, unless the internal velocities are of the order of a thousand $\mathrm{km} \mathrm{sec}^{-1}$. With such larger velocities it becomes difficult to observe the very wide lines with our $10 \mathrm{MHz}$ bandwidth.

Van Woerden: The Balmer lines are recombination lines, too. Is the fact that you do not find radio recombination lines consistent with the fact that Balmer lines are observed in such sources?

Pottasch: The answer may be that in the optical observations we're observing very thin filaments in the Balmer lines. Perhaps because of their very high relative velocity of a thousand $\mathrm{km} \mathrm{sec}^{-1}$, these velocities just smear out in the radio observations. 


\section{Various Shorter Comments}

Menon: You mention a value of about 10 per cent as being the ratio of gas to stars. You don't mean, do you, that this is a constant for all clusters?

Mezger: For all the young compact Hil regions this seems an upper limit, although in only two or three cases the limit is based on direct star counts, for example in the Orion Nebula. In all other cases, we can infer the spectral type of the exciting star from the intrinsic radio flux, as discussed before. We then make the assumption that where $\mathrm{O}$ stars are formed, stars of lower mass are formed, too, and use, in a wild extrapolation, the mass distribution function. This leads to the estimate that about 30 times the mass of $\mathrm{O}$ stars must be present in the form of lower-mass stars. This extrapolated star mass is compared to the directly measured HII mass and in all cases of young compact $\mathrm{H}$ II regions we obtain $M_{\mathrm{H}_{\mathrm{II}}} / M_{*} \lesssim 0.1$.

Van Woerden: In this 10 per cent figure you included only the gas in the $\mathrm{H}$ II region, and not that in the surrounding Hi region?

Mezger: That is correct; it only refers to the Hil gas and not to the HI gas.

Van Woerden: So, if the Hi gas has been driven away by the pressure of the hot Hil region, this might change your conclusions?

Mezger: It does not change my conclusion that, at least in the center of an OB star cluster, the efficiency of star formation is very high and that nearly all of the mass goes into the stars. However, I will not exclude the possibility that the Hil region is embedded in an Hi cloud which made the original cloud gravitationally unstable. But this would not change the conclusion that the efficiency of the formation process is extremely high in the center of the cloud, where most of the star formation is going on.

Van Woerden: You do not believe then that the Hi gas can be driven away?

Mezger: I do not object if the gas is driven away, provided that I see it observationally.

Van Woerden: Then you must not look at the surrounding shell which might be too thin for detection, but at the gas that is seen with a different velocity in front of the Hil region.

Mezger: Could we leave the question until interferometer measurements of the 21-cm line at $\mathrm{Cal}$ Tech or at Groningen really show those shells?

Sorochenko: I want to show some new experimental evidence that the ionized hydrogen in the Orion Nebula does not have a uniform distribution, but is concentrated in clouds with a high electron density $n_{e}$. This evidence, which J. J. Berulis and I have obtained (Sorochenko and Berulis, 1969) is based on observations of the hydrogen radio recombination lines. The intensity of these lines depends on the populations of the atomic levels, which in turn depend on the density. For higher densities, the population must be close to LTE. Figure 1 shows how the parameter $b_{n}^{*}$ depends on the wavelength (or equivalently on $n$ ) for various electron densities $n_{e} ; b_{n}^{*}$ is the ratio of the actual intensity of the radio line to the intensity under conditions of LTE. If there is LTE, $b_{n}^{*}=1$, and if there is no LTE, the intensity of the lines is enhanced at $\lambda \gtrsim 5 \mathrm{~cm}$ due to the partial maser effect proposed by Goldberg. At the same time, at 


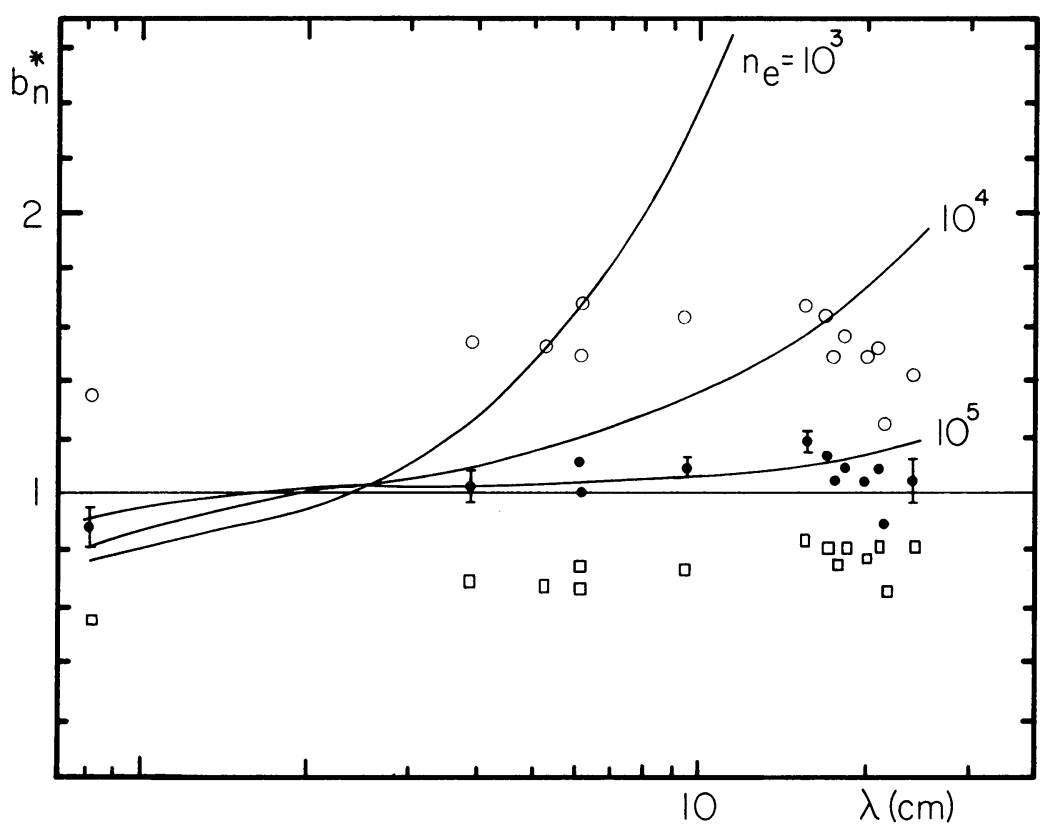

Fig. 1. (See the remark by Sorochenko.) Comparison of the theoretical and observed values of $b^{*}{ }_{n}$, where $b^{*}{ }_{n}$ is a parameter characterizing the ratio of the observed intensities of recombination lines and the predicted value for LTE. Solid lines are theoretical values for various electron densities $n_{e}$. Open circles are the observed values for $T_{e}=10^{4} \mathrm{~K}$, filled circles for $T_{e}=7 \times 10^{3} \mathrm{~K}$, and squares for $T_{e}=5 \times 10^{3} \mathrm{~K}$. This diagram summarizes results by various observers.

millimeter wavelengths there is underpopulation, and the actual intensity is lower. The three families of points correspond to observed values if we adopt electron temperatures $T_{e}=5 \times 10^{3}, 7 \times 10^{3}$, and $10^{4} \mathrm{~K}$. Good agreement of computed and measured values occurs only with $n_{e}=10^{5} \mathrm{~cm}^{-3}$ and $T_{e}=7 \times 10^{3} \mathrm{~K}$. At the same time, observations of the continuum radiation at $\lambda=8 \mathrm{~mm}$ give $n_{e}=2.7 \times 10^{3} \mathrm{~cm}^{-3}$. Only a small part of this discrepancy may be due to inaccurate values of the cross-sections. Therefore, to reconcile the two results we must conclude that dense concentrations of electrons exist. If they exist we obtain good agreement with the value of $n_{e}=5 \times 10^{4}$ $\mathrm{cm}^{-3}$ that has been mentioned by Mezger. This figure also gives the answer to the question asked earlier by Field about deviations from LTE for the radio recombination lines. For $n \gtrsim 100(\lambda \approx 5 \mathrm{~cm})$, there is no significant deviation fron LTE in the Orion Nebula; and $T_{e}=7000 \mathrm{~K}$ seems representative for $n_{e}^{2}$ averaged over the volume of the Nebula. (Sorochenko, R. L. and Berulis, J. J.: 1969, Astrophys. Lett. 4, 173).

Weliachew: I should like to draw attention to temperatures of Hil regions determined by Dr. Louise in Marseilles. He used the widths of the $\mathrm{H} \alpha$ and Nir lines. On the assumption that both lines originate in the same region, temperatures and mean turbulent velocities follow, since the atoms have different masses. The results will be published soon (Louise, 1970). [At the Symposium a table of results was passed around. (Ed.)] You will note that Louise's results are in fair agreement with the 
results from the radio recombination lines. (Louise, R.: 1970, Astron. Astrophys. 6, 460. [The following part of the discussion, up to Section 5 , actually took place on September 16 (Ed.).]

Menon: I want to comment on the observations of Harwit and Werner (1968) who claim that they observed $\mathrm{H}_{2}$ infrared radiation from a dark cloud in Orion (see Figure 2 of Mezger's Report, p. 339. The dust cloud is located at $\alpha=05^{\mathrm{h}} 33^{\mathrm{m}} 00^{\mathrm{s}}$, $\delta=-05^{\circ} 25^{\prime}$ ). I recently observed this same cloud in $21-\mathrm{cm}$ radiation, where it is seen in emission as well as in absorption. Observations with high resolution suggest that the brightness temperature of the $21-\mathrm{cm}$ radiation from that cloud is at least $60 \mathrm{~K}$. It could be that the actual kinetic temperature is higher; the line does not seem saturated. In addition, there are two interesting things. One is that the dark band is approaching the $\mathrm{HII}$ region with a velocity of about $14 \mathrm{~km} \mathrm{sec}^{-1}$. (The excess emission seen due to the band is $+12 \mathrm{~km} \mathrm{sec}^{-1}$; and the velocity of the $\mathrm{HII}$ region is $-3 \mathrm{~km} \mathrm{sec}^{-1}$, both with respect to the local standard of rest.) Of course this is only a radial motion. One end of the dark band probably has a somewhat lower velocity. This is also the region where the so-called excited line of carbon is being observed. The velocities of the carbon line (if it is carbon) and the cloud are very close to each other. This is important because the velocity observed of the order of $+12 \mathrm{~km} \mathrm{sec}^{-1}$ is essentially a peculiar velocity for this neighborhood. The density of neutral hydrogen in this cloud is about 400 or $500 \mathrm{~cm}^{-3}$. The mass, assuming that the cloud extends only over about $4^{\prime}$ (and the 21-cm observations show that it does not extend very much farther), is of the order of $10 M_{\odot}$, perhaps less. (Werner, M. W. and Harwit, M.: 1968, Astrophys. J. 154, 881).

Burke: Which instrument did you use for these observations?

Menon: They were made with both the 140-foot and the 300-foot telescopes at Green Bank. As a matter of fact, I obtained an upper limit of the brightness temperatures by looking at the excess emission. In the 140 -foot it is about $10 \mathrm{~K}$, in the 300 foot it is about $40 \mathrm{~K}$. Correcting from antenna to brightness temperature, I obtain a brightness temperature of about $60 \mathrm{~K}$.

Burke: I asked this question because I see that the angular size of this structure is about 1 or $2^{\prime}$ in declination. That is still much less than the resolution of the 300 -foot dish. How do you know that that $21-\mathrm{cm}$ cloud is associated with that dust cloud?

Menon: When you make a complete contour diagram of the $21-\mathrm{cm}$ emission, there is no question about it.

Van Woerden: Do you have an excess brightness temperature over the surroundings? I am asking this because you find $60 \mathrm{~K}$ brightness temperature at $+12 \mathrm{~km} \mathrm{sec}^{-1}$ everywhere around the Orion Nebula.

Menon: No, you do not find it in this part of the Orion region. When you take a drift curve, you find it only within one beamwidth.

Verschuur: In the very dense cloud in front of the Orion Nebula, I have found a very strong magnetic field of $50 \mu \mathrm{G}$. In my Report I suggested that these strong fields were the result of amplification in a contracting medium. The magnetic energy in this cloud, however, is about 10 to 20 times greater than the gravitational energy at 
present; therefore, the cloud could not have contracted to amplify the field. Thus there must be missing matter, and I hope that this missing matter is molecular hydrogen.

Field: If I remember correctly the density given by Werner and Harwit was about $10^{4} \mathrm{~cm}^{-3}$, about ten times greater than you see in $\mathrm{HI}$, so that it is consistent with the remark made by Verschuur.

Menon: I have no comment on the amount of molecular hydrogen. You can use the same argument Heiles (1969) used for his dark clouds (he finds discrepancies of a factor of 5 or 6), simply by saying that the absorption in that cloud is several magnitudes. I have no accurate value, because it is essentially opaque, and following Heiles' calculations, I would expect several times $8 \times 10^{20} \mathrm{~cm}^{-2}$ in the line of sight. I find about $7.5 \times 10^{20} \mathrm{~cm}^{-2}$, a discrepancy of about 5 or 6 depending on the total amount of absorption through the cloud. (Heiles, C.: 1969, Astrophys. J. 156, 493.)

\section{A New Mechanism for Star Formation}

Pikel'ner: May I turn our discussion to the question of star formation? Usually star formation is connected with gravitational instability and with fragmentation of gas due to fast cooling. Indeed, this picture works for old clusters, for instance, globular clusters. But there are problems with this mechanism. For example, stellar associations and clusters have similar stellar-mass distribution functions, although associations have much larger sizes and are less concentrated. From a theoretical point of view one would expect that the gas fragments have much larger masses during the birth of an association than during the birth of a cluster. This difference should affect noticeably the stellar-mass distribution function; but no such effect is observed. One explanation might be that the associations are concentrated during the star birth but expand later on. However, the T Tau associations are younger than $O$ associations, and in the $T$ Tau associations there have never been massive stars or supernovae and it seems unlikely that these associations have much expanded. So we conclude that some other mechanism must lead to star formation, a mechanism which is not similar to that of simple gravitational instability. I would like to suggest such a mechanism. It is connected with the general model of heating of the interstellar gas by X-rays. Let us suppose that somewhere a Rayleigh-Taylor instability develops. The gas flows down the magnetic field lines and the hydrogen density at the bottom of the flux tube is growing (see the Report by Field). When the number of hydrogen atoms $\left(N_{\mathrm{H}}\right)$ is about $10^{21} \mathrm{~cm}^{-2}$, the optical depth $\tau_{x}$ for soft X-rays responsible for the heating of the gas will be more than 1 . The absorption of $\mathrm{X}$ rays will lead to a decrease in the gas temperature at the bottom of the gas layer. (Ultraviolet radiation of stars is absorbed by dust.) Very schematically we get a twolayer system: a very cold layer at the bottom and a somewhat hotter gas layer with $\tau_{x}<1$ on top of the cold layer. This hotter layer of gas will have the usually adopted gas distribution in the $z$-direction. In the beginning the thickness of the layer of cold gas is small, and the pressure in this cold layer is determined only by the weight of the 
hotter gas layer. The temperature of the cold gas is several times less than that above. For instance, in the extended hotter layer $T \approx 50 \mathrm{~K}$ and $n_{\mathrm{H}} \approx 500 \mathrm{~cm}^{-3}$, and in the cold layer $T \approx 10 \mathrm{~K}$ and $n_{\mathrm{H}} \approx 2500 \mathrm{~cm}^{-3}$. But the gas continues to flow down. The thickness in the $z$-direction of the hotter layer remains the same, because the extra gas is added to the cold layer. Ultimately the thickness of the cold layer becomes large enough to produce self-gravitation. Then this cold layer may break into stars, due to horizontal motions. Calculating the mass of these stars one finds about 0.3 to $0.5 M_{\odot}$. This is the standard value obtained under standard conditions. We know that when star formation sets in, young stars have masses of this kind, perhaps up to $1 M_{\odot}$. After some time (about $10^{7} \mathrm{yr}$ ) the mass of the forming stars becomes higher. This increase of mass can not be explained by the older theory (the gravitational instability mechanism), which predicts that the heaviest stars should be formed first. In this new picture the mass of the forming stars is proportional to $T^{2}$, where $T$ is the temperature inside the cold layer. If we take into account the heating of the cold layer by the $T$ Tau stars, which can give $\mathrm{X}$-ray and UV emission and perhaps cosmic rays, the temperature will increase several times, perhaps from $5 \mathrm{~K}$ in the very cold layer to $10 \mathrm{~K}$ or $20 \mathrm{~K}$. The new stars will be increasingly more massive, until they begin to ionize the whole region and to stop the star formation. Another possible mechanism for gradual heating of the cold layer is to have an instability which can develop at the other side of the galactic plane. The gas falling down into the cool layer gives some pressure wave, which crosses the galactic plane and propagates further. At the other side of the plane a hill begins to grow, gas flows from this hill and opens the cold layer from the bottom. Then the layer gets heated and more massive stars can be formed. A more detailed discussion will appear elsewhere (Pikel'ner, S. B.: 1970, Astrophys. Space Sci. 7, 489).

Field: Dr. Pikel'ner, your model is based on the attenuation of X-rays by the interstellar medium. I think you would agree that we are still unclear as to whether the heating in the interstellar medium is due to cosmic rays or to X-radiation. If it is cosmic radiation, then $1 \mathrm{MeV}$ particles will have a free path just about equal to those of the X-rays that you were considering. So we would, perhaps, expect similar effects in that case. Concerning the time-dependence of star formation in a cosmological model, would not your model suggest, if it does in fact depend upon extra-galactic X-rays, that there would be a considerable dependence upon the cosmological time, since as the expansion proceeds the $\mathrm{X}$-radiation is reduced?

Pikel'ner: As for choosing between X-rays and cosmic rays, you are correct. We do not have yet definite arguments to prefer one or the other. But we have more or less reliable X-ray observations, and therefore, X-rays are somewhat preferable. The second question of cosmological time-dependence may be important. However, according to my memory, it seems at present that soft X-rays come mainly from galactic sources, but not from extra-galactic ones, because in the southern hemisphere the intensity of this flux is higher than in the northern hemisphere. In the early states of galaxy formation, the mechanism I propose was hardly important; one expects that at that time gravitational instability was more common. 
Silk: What was the X-ray flux that you assumed in getting your critical mass for star formation?

Pikel'ner: I do not assume a certain value for the X-ray flux. The only suggestion is that $\mathrm{X}$-rays heat the gas, and that $10^{21}$ atom $\mathrm{cm}^{-2}$ give a considerable absorption.

Weyman: Dr. Pikel'ner, I did not understand the figure of 0.5 to $0.3 M_{\odot}$. Was that the minimum mass, or was that supposed to be the mass-range in which the stars formed? And, if that was the case, why did you not get larger masses?

Pikel'ner: 0.3 to $0.5 M_{\odot}$ is the standard mass appearing in the thin cold layer. Star formation starts in the cold layer, when the thickness of this layer is such that its selfgravitation is the same as the external pressure. The average mass of the stars is about two times the mass of a cube with a size like the thickness of the layer (about $0.1 \mathrm{pc}$ ); the resulting mass is roughly about 0.3 to $0.5 M_{\odot}$. The stellar mass is proportional to $T^{2}$ and depends on the molecular weight. An interesting feature is that we get here stars of very low mass without any difficulties. When one tries to explain the formation of low-mass stars from globulae by gravitational instability, one finds that it is very difficult. And Kuhi (1966) quotes evidence that the more massive OB stars appear usually in $\mathrm{H}_{\mathrm{II}}$ regions containing globulae. But $\mathrm{T}$ Tau stars appear only in $\mathrm{T}$ associations. Formation of low-mass stars can occur only in a very cold gas; note that Heiles (1970) has observed such very cold regions in the OH lines. (Kuhi, L. V.: 1966, J. Roy. Astron. Soc. 60, 1 ; Heiles, C.: 1970, Astrophys. J. 160, 51.)

Weymann: You were saying earlier that it was difficult to understand why one has the same mass distribution function, even though initial conditions were different. I do not understand why this is a difficulty when the cloud masses are very much larger than the masses of the stars formed. Take, for example, the case of a turbulent spectrum. At wavelengths comparable to the scale at which energy input takes place, one expects the spectrum to depend strongly upon the details of how the energy was fed in. But at wavelengths which are very much smaller, one does not expect a strong dependence. In fact, it seems to me that we still know almost nothing about the general question of the mass spectrum for stars in various situations. I would suggest that this is one area where the aerodynamicists can, perhaps, make a really significant contribution.

Pikel'ner: I agree with your comment that a turbulence spectrum can give more or less uniform stellar-mass distribution functions, and that this function may be different under different conditions. The main point I wanted to make concerned the average mass of stars which should be larger or smaller in different conditions. 